Supplement of Geosci. Model Dev. Discuss., 8, 6867-6929, 2015

http://www.geosci-model-dev-discuss.net/8/6867/2015/

doi:10.5194/gmdd-8-6867-2015-supplement

(C) Author(s) 2015. CC Attribution 3.0 License.

(c) (i)

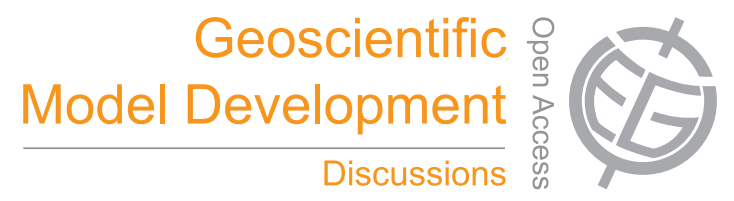

Supplement of

\title{
Validation of 3D-CMCC Forest Ecosystem Model (v.5.1) against eddy covariance data for ten European forest sites
}

\section{A. Collalti et al.}

Correspondence to: A. Collalti (alessio.collalti@cmcc.it)

The copyright of individual parts of the supplement might differ from the CC-BY 3.0 licence. 


\section{Supplementary material - Table captions}

Table S1 Performance statistics (r, NRMSE, MEF, and Bi) are reported as derived from daily and monthly series of $\mathrm{GPP}_{\mathrm{MD}}$ vs. GPP $\mathrm{GC}_{\mathrm{EC}}$ values operated over long-term annual scale. For the different indices, the values in bold represent the maximum, values in italics the minimum. The $(*)$ refers to p-value $<0.0001$ in correlation between $\mathrm{GPP}_{\mathrm{EC}}$ and $\mathrm{GPP}_{\mathrm{MD}}$ data. In addition, long term average of annual measured $\mathrm{GPP}_{\mathrm{EC}}$ and $\mathrm{GPP}_{\mathrm{MD}}$ values $\left(\mathrm{gC} \mathrm{m}^{-2} \mathrm{yr}^{-1}\right)$ are shown.

Table S2 Performance statistics (r, NRMSE, MEF, Bi) are reported as derived from daily and monthly series of $\mathrm{GPP}_{\mathrm{MD}}$ vs. $\mathrm{GPP}_{\mathrm{EC}}$ values operated over long-term seasonal scale. For the different indices, the values in bold represent the maximum, values in italics the minimum. For Bi, daily values are in $\mathrm{gC} \mathrm{m}-2 \mathrm{~d}-1$, monthly values in $\mathrm{gC} \mathrm{m}^{-2}$ month $^{-1}$. Values for DBF species in DJF are missing since the model does not produce any GPP flux during that season. The $(*)$ refers to p-value $<0.0001$ in correlation between $\mathrm{GPP}_{\mathrm{EC}}$ and $\mathrm{GPP}_{\mathrm{MD}}$ data. 
Table S1

\begin{tabular}{|c|c|c|c|c|c|c|c|c|c|c|c|c|c|c|}
\hline \multirow[b]{2}{*}{ Site Name } & \multicolumn{4}{|c|}{ DAILY } & \multicolumn{4}{|c|}{ MONTHLY } & \multicolumn{6}{|c|}{ YEARLY } \\
\hline & $r$ & NRMSE & MEF & $\begin{array}{c}B i \\
\left(g C m^{-2} d^{-1}\right)\end{array}$ & $r$ & NRMSE & MEF & $\begin{array}{c}\mathrm{Bi} \\
\left(\mathrm{gC} \mathrm{m} \mathrm{month}^{-1}\right)\end{array}$ & $r$ & NRMSE & MEF & $\begin{array}{c}\mathrm{Bi} \\
\left(\mathrm{gC} \mathrm{m}^{-2} \mathrm{yr}^{-1}\right)\end{array}$ & $\begin{array}{c}\mathrm{GPP}_{\mathrm{MD}} \\
\left(\mathrm{gC} \mathrm{m}^{-2} \mathrm{yr}^{-1}\right)\end{array}$ & 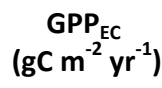 \\
\hline DE-Hai & $0.92 *$ & 0.4 & 0.85 & 0.10 & $0.97 *$ & 0.24 & 0.94 & 2.95 & 0.32 & 1.08 & -0.34 & -37.0 & 1474 & 1511 \\
\hline DK-Sor & $0.89 *$ & 0.51 & 0.74 & 0.13 & $0.97^{*}$ & 0.27 & 0.93 & 3.89 & -0.02 & 1.82 & -2.72 & -46.6 & 1901 & 1947 \\
\hline FR-Hes & $0.79 *$ & 0.77 & 0.42 & -0.85 & $0.94 *$ & 0.36 & 0.87 & -8.66 & 0.01 & 1.18 & -0.62 & 103.9 & 1726 & 1622 \\
\hline IT-Col & $0.85^{*}$ & 0.59 & 0.66 & -0.16 & $0.9 *$ & 0.46 & 0.85 & -1.9 & 0.10 & 1.15 & -0.44 & 92.6 & 1317 & 1368 \\
\hline FR-Pue & $0.82^{*}$ & 0.78 & 0.39 & -0.45 & $0.89 *$ & 0.67 & 0.58 & -13.65 & 0.76 & 1.12 & -0.38 & 157.6 & 1463 & 1305 \\
\hline IT-Cpz & $0.57^{*}$ & 1.02 & -0.04 & 0.89 & $0.71^{*}$ & 0.9 & 0.18 & 27.05 & -0.46 & 2.43 & -5.73 & -317.9 & 1409 & 1727 \\
\hline $\begin{array}{l}\text { DE-Tha } \\
\text { (1S) }\end{array}$ & $0.89 *$ & 0.48 & 0.80 & -0.08 & $0.96 *$ & 0.29 & 0.91 & -2.46 & 0.11 & 1.22 & -0.49 & 29.5 & 1898 & 1869 \\
\hline $\mathrm{FI}-\mathrm{Hyy}$ & $0.91^{*}$ & 0.48 & 0.77 & -0.14 & $0.96 *$ & 0.29 & 0.93 & -4.06 & 0.52 & 1.04 & -0.18 & 51.5 & 1119 & 1068 \\
\hline $\begin{array}{l}\text { IT-Ren } \\
\text { (2L_2C) }\end{array}$ & $0.81^{*}$ & 0.62 & 0.61 & -0.02 & $0.95^{*}$ & 0.3 & 0.91 & -0.72 & -0.47 & 1.43 & -1.45 & -14 & 1348 & 1362 \\
\hline $\begin{array}{c}\text { BE-Bra } \\
\text { (P_Q-3L) }\end{array}$ & $0.77^{*}$ & 0.64 & 0.58 & 0.18 & $0.91 *$ & 0.42 & 0.82 & -3.95 & -0.38 & 1.23 & -0.69 & -57 & 1169 & 1112 \\
\hline Average & 0.83 & 0.63 & 0.58 & $|0.30|$ & 0.92 & 0.42 & 0.79 & |6.93| & - & 1.37 & -1.30 & |90.8| & 1482 & 1489 \\
\hline
\end{tabular}


Table S2

\begin{tabular}{|c|c|c|c|c|c|c|c|c|c|c|c|c|c|c|c|c|}
\hline \multicolumn{17}{|c|}{ MONTHLY } \\
\hline \multirow{2}{*}{ Sites } & \multicolumn{4}{|c|}{ DJF } & \multicolumn{4}{|c|}{ MAM } & \multicolumn{4}{|c|}{ JJA } & \multicolumn{4}{|c|}{ SON } \\
\hline & $r$ & NRMSE & MEF & $\mathrm{Bi}$ & $r$ & NRMSE & MEF & $\mathrm{Bi}$ & $r$ & NRMSE & MEF & $\mathrm{Bi}$ & $r$ & NRMSE & MEF & $\mathrm{Bi}$ \\
\hline DE-Hai & - & - & - & - & $0.98^{*}$ & 0.48 & 0.87 & -21.31 & 0.64 & 1.02 & -0.42 & 28.62 & $0.99 *$ & 0.17 & 0.97 & 4.53 \\
\hline DK-Sor & - & - & - & - & $0.95^{*}$ & 0.45 & 0.85 & 29.88 & $0.77^{*}$ & 0.64 & 0.28 & -8.88 & $0.97^{*}$ & 0.46 & 0.88 & -11.98 \\
\hline FR-Hes & - & - & - & - & $0.97^{*}$ & 0.24 & 0.94 & 0.85 & 0.32 & 0.99 & -2.13 & -20.56 & $0.9^{*}$ & 0.57 & 0.77 & -18.91 \\
\hline IT-Col & - & - & - & - & $0.77^{*}$ & 0.72 & 0.52 & 19.35 & 0.27 & 1.13 & -0.81 & -12.43 & $0.71^{*}$ & 0.78 & 0.36 & -19.69 \\
\hline FR-Pue & $0.79 *$ & 1.2 & -0.12 & 12.82 & $0.81 *$ & 1.19 & 0.18 & -28.66 & $0.83 *$ & 0.81 & 0.02 & -36.44 & $0.74 *$ & 0.84 & 0.53 & -2.72 \\
\hline IT-Cpz & 0.05 & 1.56 & -8.95 & 37.75 & 0.06 & 1.49 & -0.63 & 6.94 & 0.5 & 1.07 & -1.84 & 29.69 & 0.58 & 1.15 & -1.39 & 33.82 \\
\hline $\begin{array}{l}\text { DE-Tha } \\
\text { (1S) }\end{array}$ & $0.88^{*}$ & 0.5 & 0.73 & -1.88 & $0.89 *$ & 0.52 & 0.75 & -19.5 & 0.57 & 0.88 & -0.35 & 13.35 & $0.95^{*}$ & 0.38 & 0.89 & -1.81 \\
\hline $\mathrm{FI}-\mathrm{Hyy}$ & $0.71^{*}$ & 0.94 & -127 & 1.16 & $0.96 *$ & 0.63 & 0.76 & -27.79 & 0.62 & 0.99 & 0.3 & -7.32 & $0.96 *$ & 0.49 & 0.69 & 18.56 \\
\hline $\begin{array}{c}\text { IT- } \\
\text { Ren(2L- } \\
2 \mathrm{C})\end{array}$ & 0.39 & 1.15 & -0.54 & -5.95 & $0.88^{*}$ & 0.42 & 0.79 & 3.44 & 0.69 & 1 & -1.92 & -10.32 & $0.94 *$ & 0.47 & 0.76 & 15.68 \\
\hline $\begin{array}{c}\text { BE-Bra } \\
\text { (P_Q-3L) }\end{array}$ & 0.39 & 1.79 & -0.81 & -9.72 & $0.91^{*}$ & 0.58 & 0.46 & -23.02 & 0.08 & 1.12 & -5.3 & 17.8 & $0.87^{*}$ & 0.48 & 0.68 & -1.08 \\
\hline Average & 0.54 & 1.19 & -22.78 & 5.70 & 0.82 & 0.67 & 0.55 & -5.98 & 0.53 & 0.97 & -1.22 & -0.65 & 0.86 & 0.58 & 0.51 & 1.64 \\
\hline \multicolumn{17}{|c|}{ DAILY } \\
\hline DE-Hai & - & - & - & - & $0.88^{*}$ & 0.81 & 0.7 & -0.7 & $0.74 *$ & 0.91 & 0.46 & 0.93 & $0.94 *$ & 0.36 & 0.88 & 0.15 \\
\hline DK-Sor & - & - & - & - & $0.85^{*}$ & 0.73 & 0.7 & 0.83 & $0.48^{*}$ & 1.12 & 0.09 & -0.23 & $0.87^{*}$ & 0.7 & 0.73 & -0.3 \\
\hline
\end{tabular}




\begin{tabular}{|c|c|c|c|c|c|c|c|c|c|c|c|c|c|c|c|c|}
\hline FR-Hes & - & - & - & - & $0.77^{*}$ & 0.84 & 0.58 & -0.55 & $0.17^{*}$ & 1.36 & -0.95 & -1.77 & $0.71 *$ & 1.06 & 0.41 & -1.18 \\
\hline IT-Col & - & - & - & - & $0.69 *$ & 0.87 & 0.43 & 0.55 & $0.42 *$ & 1.18 & -0.03 & -0.44 & $0.72 *$ & 0.83 & 0.43 & -0.79 \\
\hline FR-Pue & $0.84 *$ & 0.77 & 0.55 & 0.42 & $0.79 *$ & 1.12 & 0.48 & -0.93 & $0.72 *$ & 0.9 & 0.15 & -1.18 & $0.73 *$ & 0.81 & 0.52 & -0.09 \\
\hline IT-Cpz & $0.21^{*}$ & 1.38 & -3.45 & 1.27 & $0.16^{*}$ & 1.41 & -0.47 & 0.19 & $0.36 *$ & 1.15 & -1.12 & 0.99 & $0.46^{*}$ & 1.16 & -1.03 & 1.14 \\
\hline $\begin{array}{l}\text { DE-Tha } \\
\text { (1S) }\end{array}$ & $0.75^{*}$ & 0.85 & 0.55 & -0.07 & $0.82 *$ & 0.74 & 0.65 & -0.64 & $0.63^{*}$ & 0.92 & 0.31 & 0.44 & $0.89 *$ & 0.57 & 0.79 & -0.06 \\
\hline Fl-Hyy & $0.35^{*}$ & 0.96 & -33.28 & 0.04 & $0.86^{*}$ & 0.94 & 0.62 & -0.91 & $0.73 *$ & 1.07 & 0.52 & -0.24 & $0.91 *$ & 0.53 & 0.7 & 0.59 \\
\hline $\begin{array}{c}\text { IT- } \\
\text { Ren(2L- } \\
2 \mathrm{C})\end{array}$ & $0.23^{*}$ & 1.29 & -0.6 & -0.2 & $0.73^{*}$ & 0.77 & 0.5 & 0.07 & $0.31 *$ & 1.27 & -0.56 & -0.34 & $0.73 *$ & 0.79 & 0.4 & 0.57 \\
\hline $\begin{array}{c}\text { BE-Bra } \\
\text { (P_Q-3L) }\end{array}$ & $0.38^{*}$ & 1.03 & -1.09 & -0.23 & $0.68^{*}$ & 0.86 & 0.28 & -0.6 & $0.19^{*}$ & 1.35 & -1.24 & 1.25 & $0.69^{*}$ & 0.74 & 0.2 & 0.18 \\
\hline Average & 0.46 & 1.05 & -6.22 & 0.21 & 0.72 & 0.91 & 0.45 & -0.27 & 0.48 & 1.12 & -0.24 & -0.06 & 0.77 & 0.76 & 0.40 & 0.02 \\
\hline
\end{tabular}



